\title{
Erratum to: Big Data Processing for Renewable Energy Telemetry Using a Decentralized Cloud M2M System
}

\author{
George Suciu' ${ }^{1}$ Alexandru Vulpe ${ }^{1}$. \\ Alexandru Martian ${ }^{1} \cdot$ Simona Halunga ${ }^{1}$. \\ Dragos Nicolae Vizireanu ${ }^{1}$
}

Published online: 12 May 2015

(C) Springer Science+Business Media New York 2015

\section{Erratum to: Wireless Pers Commun DOI 10.1007/s11277-015-2527-7}

The following Financial Agreement was omitted in the Acknowledgments section of the original publication: POSDRU/159/1.5/S/132395. The complete, correct Acknowledgments section is shown below.

Acknowledgments This work has been funded by the Sectoral Operational Programme Human Resources Development 2007-2013 of the Ministry of European Funds through the Financial Agreements POSDRU/ 159/1.5/S/134398 and POSDRU/159/1.5/S/132395, and supported by UEFISCDI Romania, under the Grant No. 20/2012 "SaRaT-IWSN". The authors are grateful to Adelina Ochian and Laura Ghenciu from Beia Consult International for the fruitful discussions that helped in the writing of this paper.

The online version of the original article can be found under doi:10.1007/s11277-015-2527-7.

George Suciu

george@beia.ro

Alexandru Vulpe

alex.vulpe@radio.pub.ro

Alexandru Martian

martian@radio.pub.ro

Simona Halunga

shalunga@elcom.pub.ro

Dragos Nicolae Vizireanu

nvizireanu@comm.pub.ro

1 Faculty of Electronics, Telecommunications and Information Technology, University Politehnica of Bucharest, 060071 Bucharest-6, Romania 